\title{
A novel technique to achieve cutaneous continent urinary diversion in spinal cord-injured patients unable to catheterize through native urethra
}

\author{
G Karsenty ${ }^{1}$, E Chartier-Kastler ${ }^{1}$, P Mozer ${ }^{1}$, A Even-Schneider ${ }^{2}$, P Denys ${ }^{2}$ and F Richard ${ }^{1}$ \\ ${ }^{1}$ Department of Urology, Pitié-Salpetrière Hospital, Pierre et Marie Curie School of Medicine (Paris VI), Paris, France and ${ }^{2}$ Department \\ of Physical Medicine and Rehabilitation, Garches Hospital, Paris Ouest School of Medicine (University Versailles Saint Quentin-en- \\ Yvelines), Boulogne, France
}

\begin{abstract}
Study design: Description of a technique and prospective follow-up study.
Objectives: To present and assess a perioperative strategy associated with a single-procedure surgical technique for continent cutaneous diversion in spinal cord injury ( $\mathrm{SCl}$ ) patients requiring selfcatheterization and unable to do it through the native urethra.

Setting: University hospital, Paris, France.

Methods: We considered $\mathrm{SCl}$ patients suffering from urinary incontinence related to neurogenic detrusor overactivity and/or poor bladder emptying for more than 1 year and inability to perform selfcatheterization through the native urethra. These patients including quadriplegics underwent selection for surgery by occupational therapists and neurorehabs to assess the ability to self catheterize through an abdominal stoma and to determine the optimal site to place the stoma. The surgical technique included a single procedure: aponevrotic sling in women requiring stress continence reinforcement, supratrigonal cystectomy, preserved detrusor wall flap (original description), enterocystoplasty, a catheterizable tube using either the Mitrofanoff or Young-Monti principle and Politano-Leadbetter antireflux technique. A prospective follow-up study of consecutive patients reviewed initial condition, indication, surgical technique, complications, continence, catheterizing difficulties, functional bladder capacity and serum creatinine.

Results: Thirteen consecutive patients were selected for surgery. Median follow-up was 44 months. Stoma location was variable from one patient to another. All patients had a catheterizable continent stoma at last follow-up. Kidney function was preserved.

Conclusion: Given these results, a multidisciplinary approach including neuro-rehabilitation practitioners and urologists performing appropriate technical solutions in highly selected $\mathrm{SCl}$ patients unable to catheterize native urethra provides upper urinary tract protection and continence after a single procedure.
\end{abstract}

Spinal Cord (2008) 46, 305-310; doi:10.1038/sj.sc.3102104; published online 14 August 2007

Keywords: spinal cord injury; quadriplegia; urinary diversion; incontinence; urinary catheterization; urinary bladder

\section{Introduction}

Spinal cord lesions induce severe dysfunction of the bladder and the urethral sphincter, which impair both micturition and urine storage. ${ }^{1}$ Urological management aims to prevent life-threatening complications, and to improve patient autonomy and quality of life. ${ }^{2}$ Clean intermittent self-catheterization has been demonstrated to

Correspondence: Dr G Karsenty, Department of Urology, Pitié-Salpetrière Hospital, Pierre et Marie Curie School of Medicine (Paris VI), $47 \mathrm{Bd}$ de I'Hôpital, 75013 Paris, France.

E-mail: gilles.karsenty@ap-hm.fr

Received 4 February 2007; revised 29 May 2007; accepted 3 June 2007; published online 14 August 2007 be the most-effective way to empty the bladder in spinal cord injury (SCI) patients. To prevent upper urinary tract damage and achieve continence, one must obtain a low-pressure bladder of adequate volume. When validated conservative treatment of neurogenic detrusor overactivity fails, enterocystoplasty (ECP) has been demonstrated to be a safe and effective alternative. ${ }^{3,4}$

However, urethral catheterization can be impossible or unacceptable in some SCI patients. Upper limb dysfunction, difficulty in transferring or undressing oneself, inability to reach the urethra, or urethral lesions are the usual reasons that an SCI patient is unable to catheterize. In such 
situations, a cutaneous continent diversion (CCD) composed of an abdominal continent stoma (ACS) combined with ECP can facilitate self-catheterization.

We describe and analyze our perioperative strategy and technique for CCD in SCI patients requiring self-catheterization and unable to do it through the native urethra.

\section{Patients and methods}

\section{Patient selection}

ECP combined with ACS was considered in selected SCI patients with urinary incontinence related to neurogenic detrusor overactivity, and/or poor bladder emptying for over 1 year.

Selection criteria in paraplegic patients included poor bladder emptying owing to detrusor sphincter dyssynergia or bladder acontractility and inability to perform selfcatheterization through the native urethra.

Selection criteria in male quadriplegic patients included poor bladder emptying and inability to perform selfcatheterization through the native urethra and refusal of sphincterotomy (endoscopic surgery or urethral stent) or Brindley neurostimulator. Sphincterotomy is never considered in tetraplegic women. In both genders, a creatinin clearance lower than $40 \mathrm{ml} / \mathrm{min}$ was considered as a contraindication because of increased risk of morbid hyperchloremic acidosis due to ionic reabsorption through the intestinal mucosa of a continent urinary pouch. ${ }^{5}$ Selected patients were evaluated for both motivation and the ability to selfcatheterize through an abdominal stoma while sitting. Patients were asked to catheterize a fake stoma on the lower abdominal wall. Optimal stoma location was chosen according to the time and ease of self-catheterization. Time to handle the catheter as well as to undress and dress up again was taken into account. Motivation was evaluated with the patient, family and occupational therapist only after 1 week of such trial. Patients who successfully completed this evaluation were offered surgery.

\section{Surgical technique}

In female patients with mixed urinary incontinence, open bladder neck or low maximum urethral pressure (MU$\mathrm{P}<30 \mathrm{~cm} \mathrm{H}_{2} \mathrm{O}$ ), a bladder neck aponeurotic sling anchored to Cooper's ligaments was first performed to reinforce stress continence mechanisms (Figure 1). Supratrigonal cystectomy to remove pathological detrusor was performed first in men. Ureteric stents were placed at the time of the surgery and removed before augmented bladder closure. A long posterolateral bladder wall flap of $2.5-3 \mathrm{~cm}$ width and full wall thickness was preserved (Figure 2). Coloration and bleeding from the distal part of the flap were carefully verified to detect local ischemia before the catheterizable tube was implanted in the flap. A maximal flap length of $16.5 \mathrm{~cm}$ was obtained. The flap was preserved from the right or left hemi-bladder according to the location of the stoma. In this patient series, all flaps came from the right hemibladder. The bladder neck and urethra were left open to preserve a pop-off mechanism and for easy access to the

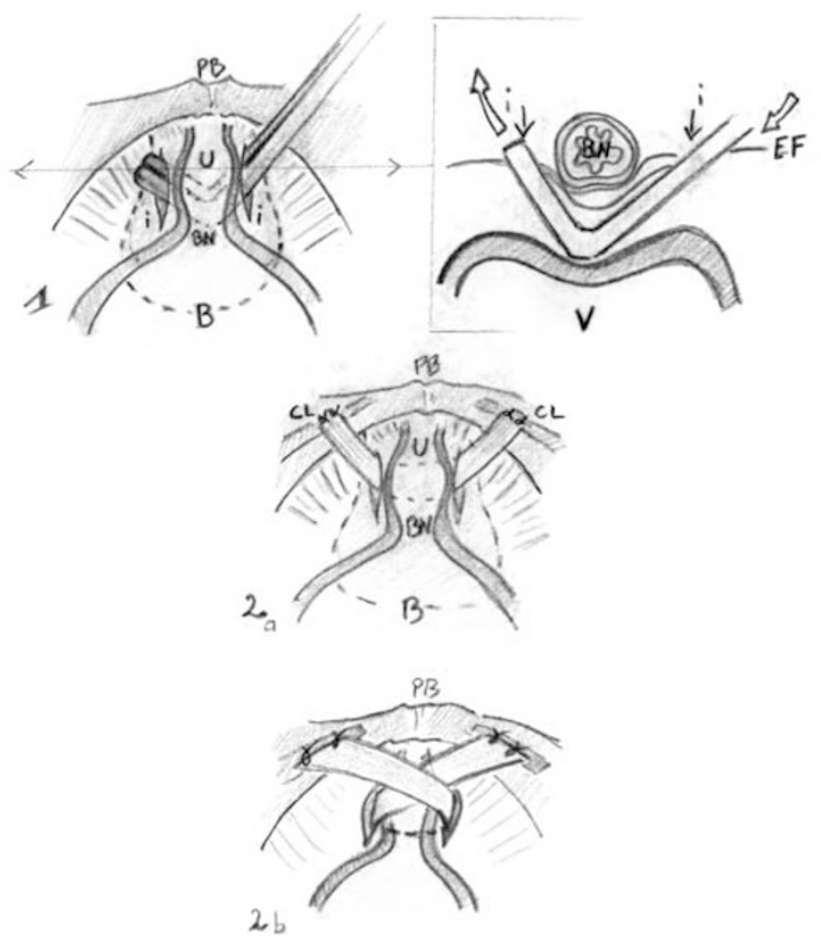

Figure 1 Reinforced stress continence mechanism in women. Aponeurotic sling anchored to Cooper's ligament supports the bladder neck to reinforce stress continence mechanisms.

bladder in case of catheterization difficulty. The excised part of the bladder was replaced by a $55-\mathrm{cm}$ ileal segment $30 \mathrm{~cm}$ from the ileo-caecal valve, detubularized according to Hautmann's technique, ${ }^{6}$ then anastomosed to the trigone. The efferent catheterizable tube was constructed using either the Mitrofanoff $^{7}$ or Yang-Monti ${ }^{8}$ principle according to surgical history, appendix length and patency (Figure 3). The capacity of the efferent tube to reach the planned stoma site without tension was verified at this time. The proximal efferent tube was implanted into the detrusor flap using the Politano-Leadbetter ${ }^{9}$ anti-reflux technique (Figure 2). The absence of kink and the direct route of catheterization were verified before the efferent tube was sutured to the skin with a V-shaped skin flap. A 14-French Foley catheter was left in the efferent tube and a 20- or 22-French urethral catheter was placed to drain the ECP (dedicated to ECP with large holes and additional holes bellow the balloon). Closure of the peritoneal sheet maintained the augmented bladder and efferent tube close to the inner face of the abdominal wall outside the peritoneum.

\section{Peri-operative care}

All patients were admitted $48 \mathrm{~h}$ before the procedure to receive two enemas and 21 of an oral polyethylene glycol solution.

Naso-gastric tube was removed when bowel movements resumed and then oral feeding was slowly re-introduced. Early post-operative mobilization (median: third day) was implemented to promote faster intestinal transit 


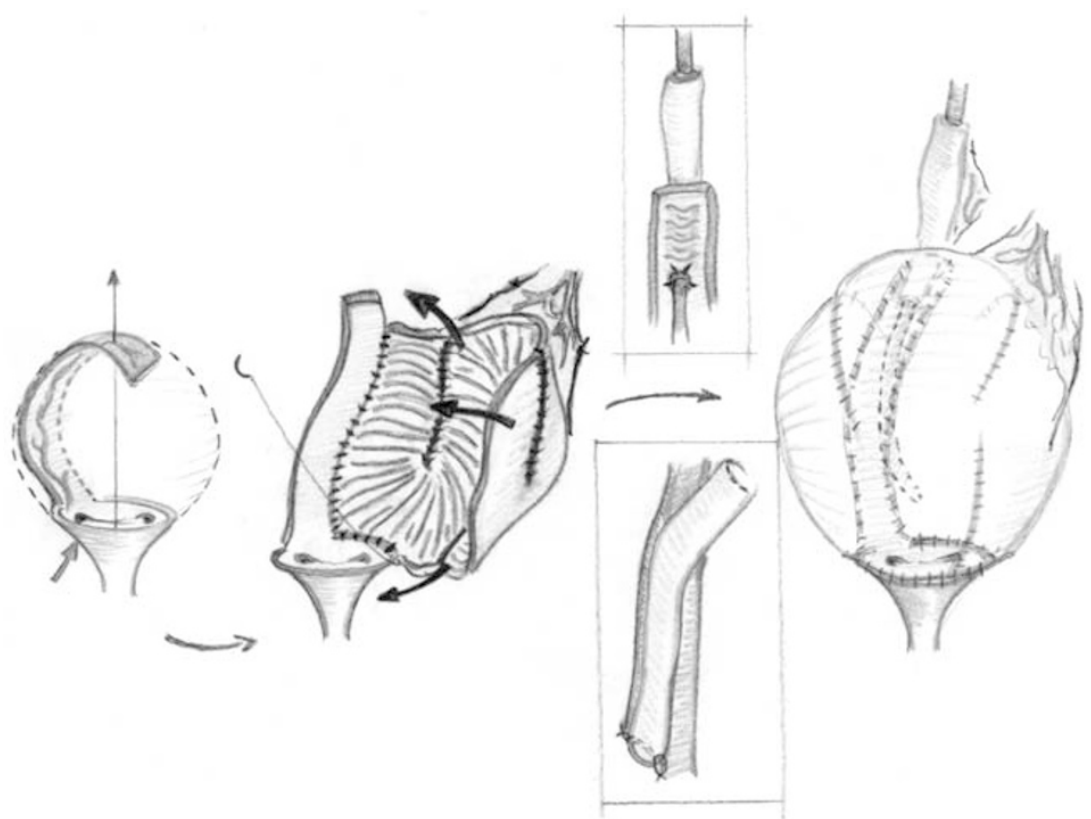

Figure 2 Enterocystoplasty (ECP) combined with abdominal continent stoma (ACS). Supratrigonal cystectomy sparing a long posterolateral bladder wall flap that respects bladder blood supply. The mobile part of the bladder is replaced by a $55-\mathrm{cm}$ ileal segment detubularized according to Hautmann's technique. Implantation of the proximal efferent tube into the detrusor flap using the Politano-Leadbetter technique (from left to right).

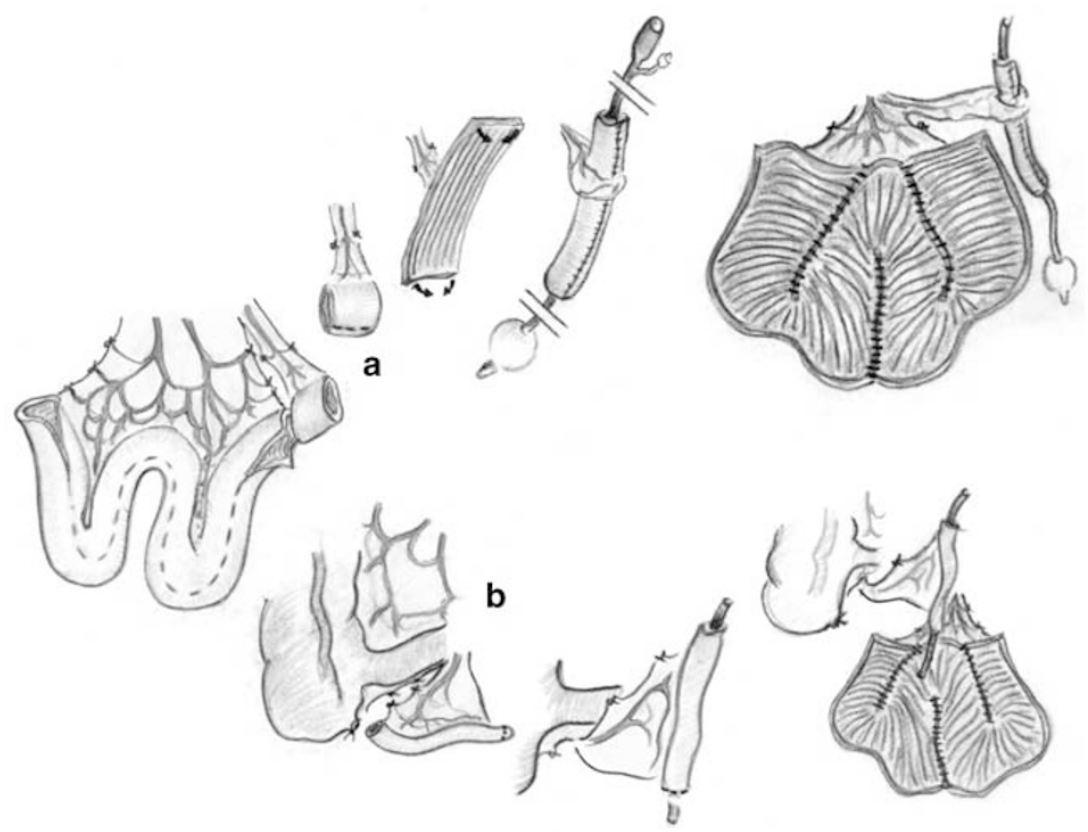

Figure 3 Technical principles of Monti (a) and Mitrofanoff tubes (b).

resumption. Saline irrigation through urethral and stomal catheters started postoperatively (three times a day) to prevent mucus build up. The urethral catheter was removed 2 days before starting self-catheterization. Self-catheterization education started progressively after 14-French catheter removal at the 21st day and was optimized at the neurorehabilitation department using the urinary diary to monitor time between catheterization (initially $3 \mathrm{~h}$ ). Saline irrigation, twice a week, was maintained during 8 weeks.

\section{Results}

Thirteen consecutive SCI patients (11 females, 2 males), underwent ACS combined with ECP from July 2001 to January 2004. Patient characteristics and indications for surgery are summarized in Table 1.

All patients had a preserved posterolateral detrusor flap. The efferent tube was constructed according to Monti in six cases and according to Mitrofanoff in seven cases. All were 
Table 1 Patient characteristics

Number of patients
Male
Female
Median age at surgery
(min-max)
Median duration of $\mathrm{SCl}$
(min-max)
Level and etiology of $\mathrm{SCl}$

Bladder dysfunction

Indication for $\mathrm{CCD}^{\mathrm{a}}$

Median BMI (min-max) Median creatinine at baseline (min-max) Upper urinary tract lesion

13

11

$42(18-63)$

12.2 years $(1.6-32)$

Cervical: 5 (C5 ASIA B-C7 ASIA A),

traumatic (3), myelitis (2)

Thoracic: 5 (T6-T10), traumatic (4), myelitis (1)

Sacral: 1 (conus medularis), traumatic

Cauda Equina: 1, traumatic

Other: 1 multiple sclerosis 10 years

without progression

$\mathrm{NDOI}+$ DSD 12

Acontractile bladder 1

Upper limb disability 6

Inability to reach or find urethra 4 (all women)

Urethral lesion (fistula \pm stenosis) 4

$20 \mathrm{~kg} / \mathrm{m}^{2}(17-27)$

$52 \mu \mathrm{mol} / /(38-97)$

One patient (grade 2 hydronephrosis) Six had more than three pyelonephritis

${ }^{a}$ One patient had urethral stenosis after post-traumatic urethral fistula and upper limb inability due to brachial plexus lesion resulting in a monoplegia. Abbreviations: $\mathrm{BMI}$, body mass index; $\mathrm{CCD}$, cutaneous continent diversion; $\mathrm{DSD}$, detrusor sphincter dyssynergia; NDOI, neurogenic detrusor overactivity incontinence; $\mathrm{SCl}$, spinal cord injury.

re-implanted in the detrusor flap according to PolitanoLeadbetter. Stoma were made at the umbilicus (7), on the midline between umbilicus and pubis (2), on the right iliac fossa (2) or in a right paraumbilical site (1). All patients had stoma at the site proposed before surgery. All female patients except one had a bladder neck sling. The duration of surgery was 250-386 min (median $355 \mathrm{~min}$ ) and blood loss was $150-1200 \mathrm{ml}$ (median $386 \mathrm{ml}$ ).

Median follow-up was 44 months (range 20-56) and one patient was lost to follow-up.

There were no postoperative deaths. One patient was reoperated for pelvic abscess and another underwent small bowel resection for occlusion 4 months after surgery. Seven patients had documented postoperative infection (five urinary, two pulmonary), which were all successfully treated with antibiotics.

At last follow-up, the catheterizable stoma was continent in all 12 patients. All patients were performing 4-6 selfcatheterizations a day on voiding diary. One patient initially had difficulty in catheterizing, but finally succeeded with a different catheter (bend tip). Three women had stress urine leakage through the native urethra despite a large bladder capacity. One was treated with a peri-urethral bulking agent, and the remaining two required secondary bladder neck closure.

Median functional bladder capacity was $180 \mathrm{ml}$ (range: $70-445 \mathrm{ml}$ ) and $540 \mathrm{ml}$ (range: $380-800 \mathrm{ml}$ ) before surgery and at last follow-up, respectively. There was no change in serum creatinine level or in renal ultrasound after surgery.

\section{Discussion}

This comprehensive technique of urinary diversion preserved kidney function and provided a catheterizable and continent stoma in all patients at last follow-up. There were no specific re-operation or self-catheterization problems. Continent lower urinary tract reconstruction in SCI patients who require bladder augmentation and cannot perform selfcatheterization via the native urethra has been reported to be effective in protecting the upper urinary tract, and enhances patient independence, self-esteem and quality of life. ${ }^{10-14}$ In accordance with Zommick et al., ${ }^{14}$ we consider preoperative selection of patients to be the key point for successful outcomes. Patients were selected based on their performance and satisfaction in self-catheterization in a fake stoma positioned at different sites on the abdomen wall. We found that the optimal stoma site was patient-specific and not always at the umbilicus. Moreover, wheelchair-bound patients often need higher placement of the stoma. An efferent tube to connect the augmented bladder to any site of the lower abdomen without length limitation was therefore necessary.

The posterolateral detrusor flap first described herein provided enough length for a single efferent tube to reach the site chosen for the stoma preoperatively in all cases. This flap appears to be very useful owing to the pelvic bone rotation related to SCI, increasing the anatomical distance from pubic bone to the ombilic by 1.5-2 times.

Although none of our patients was obese (median body mass index (BMI) $20 \mathrm{~kg} / \mathrm{m}^{2}$ ), overweight and obesity are frequent among SCI patients. ${ }^{15}$ The thickness of the abdominal wall and short, fat mesentery could restrict the feasibility of this technique in obese patients. Therefore, BMI or better waist circumference, which is thought to be a more accurate visceral adiposity predictor, ${ }^{16}$ should be taken into account in the eligibility checklist for surgery.

A double Monti tube, implanted in the detrusor flap, would make it possible to reach any point of the lower abdominal wall in those patients. Shifting the small bowel opening away from the anti-mesenteric line also shifts the location of mesentery insertion in the re-tubularized segment and provides a longer mesentery-free part at both ends of the double tube (Figure 4). The tube is therefore easier to re-implant in its proximal end and to pass through the abdominal wall in its distal end. The absence of tension and compression on the mesentery reduce the chance to compromise tube blood supply. According to the report of higher complication rate with double Monti ${ }^{17}$ (stenosis at the anastomosis of the two hemi-tubes and difficulty to catheterize), we believe that if a single tube was too short, the modification of the double Monti without anastomosis proposed by Casale $^{18}$ (Figures $5 \mathrm{a}$ and $\mathrm{b}$ ) would be the alternative choice.

Although the continence of such reservoirs largely depends on their physical qualities (capacity and compliance), re-implanting the tube according to the Politano-Leadbetter anti-reflux technique avoids urine leakage. Re-implanting the efferent tube in a preserved piece of bladder wall avoids implantation in the small bowel and provides a strong and 


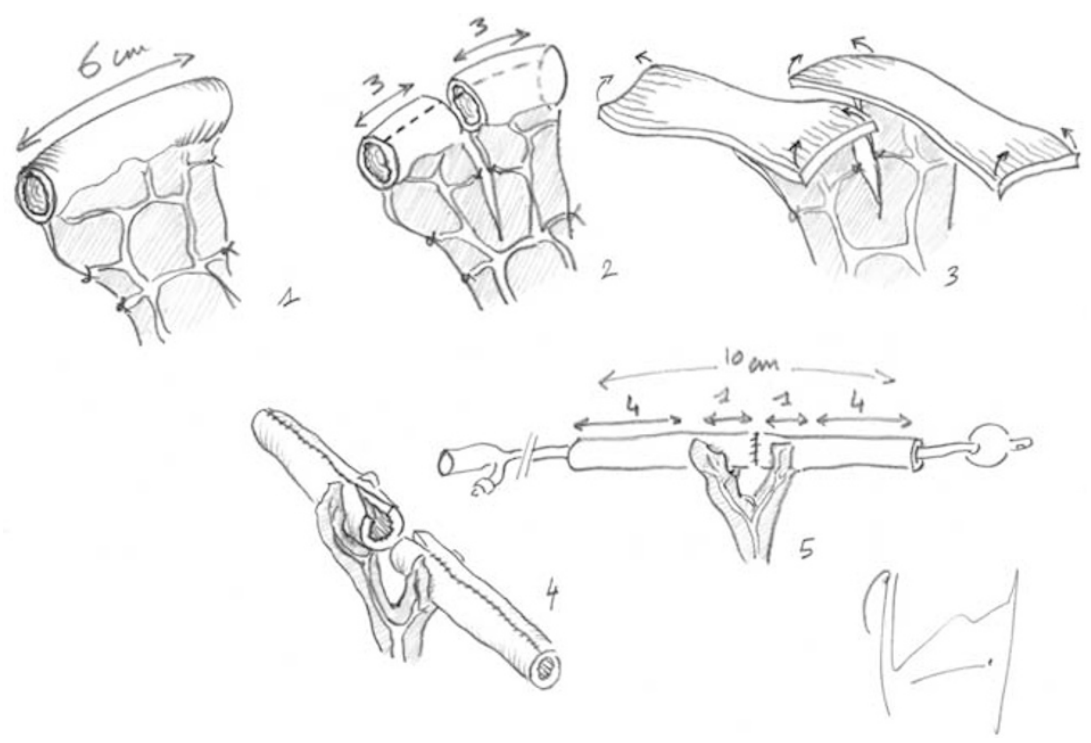

Figure 4 Technical principles of double Monti tube.

a

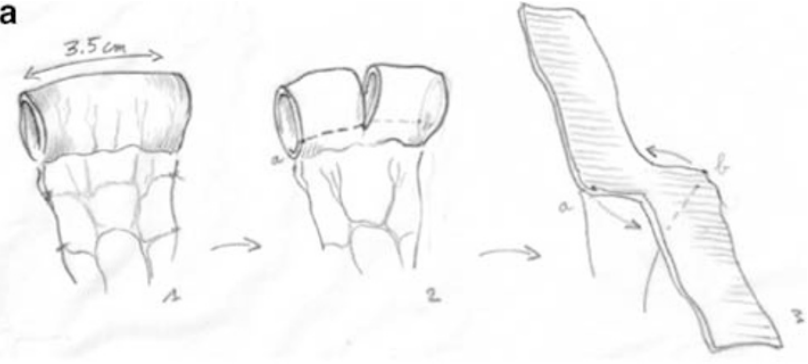

b

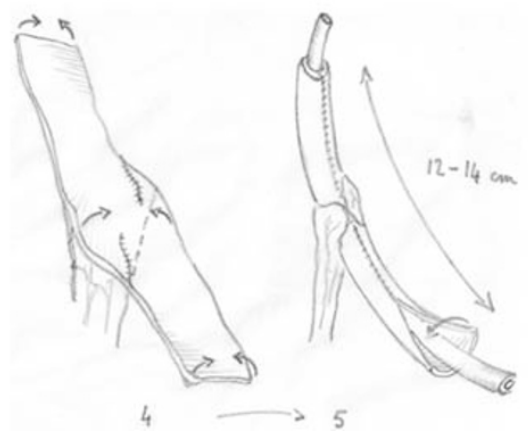

Figure 5 (a) and (b) Technical principles of Casale tube.

resistant posterior wall for self-catheterization. Whether or not the nature of the tube (appendix or detubularized ileum) affects the frequency of stoma stenosis remains controversial. ${ }^{19}$ We had no cases of severe stenosis requiring surgical revision with either tube. This could be explained by the systematic use of a V-shaped skin flap to enlarge the circumference of the stoma. The absence of tension on the tube avoiding local ischemia at its extremities could also play a role in avoiding stenosis. However, the rate of stenosis should be re-evaluated with longer follow-up and more patients.

Supratrigonal cystectomy was performed owing to the poor quality of the bladder wall observed at the time of surgery in all of our patients following the long period between spinal lesion and surgery. We believe that leaving such tissue could decrease compliance of the augmented bladder. This choice and the choice of an ileal detubularized segment to build the ECP were supported by the long-term successful outcomes we obtained with this technique in SCI patients. ${ }^{3}$

All except one of our female patients had a history of prolonged indwelling urethral catheter. Subsequent urethral and bladder neck damage could explain the frequency of mixed urinary incontinence, low MUP or the opening bladder neck we observed. As recently reported by Castellan et al., ${ }^{20}$ we associated a bladder neck fascial sling to prevent the persistence of the stress component of urinary incontinence through the native urethra. Despite this procedure, three patients required additional treatment for persistent stress urinary incontinence by the native urethra. Interestingly, they had the lowest preoperative MUP of the series and $2 / 3$ had an opened bladder neck. Castellan et al. ${ }^{20}$ also reported seven patients with mixed neurogenic incontinence that failed after bladder neck sling associated with bladder augmentation. Continence of native urethra remains a challenging situation in female para- or quadriplegic patients, especially with a long-standing indwelling catheter. In these women with risk factors of urethral leakage, the risk/ benefit ratio of an ECP combined with ACS that includes the chance of re-interventions for bladder neck closure has to be carefully assessed. Heterotopic continent diversion bearing the potentially severe long-term morbidity of ureterodigestive re-implantation ${ }^{21}$ can be discussed in such cases.

In this patient series, morbidity was acceptable ${ }^{22}$ and an increase in bladder capacity and ability to empty the bladder was achieved in 12 patients, including 5 quadriplegics. Although improvement in quality of life was not evaluated with specific tools, patients reported satisfaction and improved independence.

Such results support that an association of appropriate surgical technical solutions in carefully selected SCI patients 
can provide upper urinary tract protection, continence and a high degree of independence regarding lower urinary tract function after conservative options have failed. To meet patients' expectations and reach physicians goals, this comprehensive strategy is based on a multidisciplinary approach involving urologist and neuro-rehabilitation practitioners.

\section{References}

1 de Groat WC. Mechanisms underlying the recovery of lower urinary tract function following spinal cord injury. Paraplegia 1995; 33: 493-505.

2 Elliott DS, Boone TB. Recent advances in the management of the neurogenic bladder. Urology 2000; 56: 76-81.

3 Chartier-Kastler EJ, Mongiat-Artus P, Bitker MO, Chancellor MB, Richard F, Denys P. Long-term results of augmentation cystoplasty in spinal cord injury patients. Spinal Cord 2000; 38: 490-494.

4 Herschorn S, Hewitt RJ. Patient perspective of long-term outcome of augmentation cystoplasty for neurogenic bladder. Urology 1998; 52: 672-678.

5 Mills RD, Studer UE. Metabolic consequences of continent urinary diversion. J Urol 1999; 161: 1057-1066.

6 Hautmann RE, Egghart G, Frohneberg D, Miller K. [The ileal neobladder]. Urol A 1987; 26: 67-73.

7 Mitrofanoff P. [Trans-appendicular continent cystostomy in the management of the neurogenic bladder]. Chir Pediatr 1980; 21: 297-305.

8 Castellan MA, Gosalbez Jr R, Labbie A, Monti PR. Clinical applications of the Monti procedure as a continent catheterizable stoma. Urology 1999; 54: 152-156.

9 Politano VA, Leadbetter WF. An operative technique for the correction of vesicoureteral reflux. J Urol 1958; 167: 1055-1061; discussion 1062, 2002.
10 Hakenberg OW, Ebermayer J, Manseck A, Wirth MP. Application of the Mitrofanoff principle for intermittent self-catheterization in quadriplegic patients. Urology 2001; 58: 38-42.

11 Moreno JG, Chancellor MB, Karasick S, King S, Abdill CK, Rivas DA. Improved quality of life and sexuality with continent urinary diversion in quadriplegic women with umbilical stoma. Arch Phys Med Rehabil 1995; 76: 758-762.

12 Sutton MA, Hinson JL, Nickell KG, Boone TB. Continent ileocecal augmentation cystoplasty. Spinal Cord 1998; 36: 246-251.

13 Sylora JA, Gonzalez R, Vaughn M, Reinberg Y. Intermittent selfcatheterization by quadriplegic patients via a catheterizable Mitrofanoff channel. J Urol 1997; 157: 48-50.

14 Zommick JN, Simoneau AR, Skinner DG, Ginsberg DA. Continent lower urinary tract reconstruction in the cervical spinal cord injured population. J Urol 2003; 169: 2184-2187.

15 Gupta N, White KT, Sandford PR. Body mass index in spinal cord injury-a retrospective study. Spinal Cord 2005; 44: 92-94.

16 Buchholz AC, Bugaresti JM. A review of body mass index and waist circumference as markers of obesity and coronary heart disease risk in persons with chronic spinal cord injury. Spinal Cord 2005; 43: 513-518.

17 Narayanaswamy B, Wilcox DT, Cuckow PM, Duffy PG, Ransley PG. The Yang-Monti ileovesicostomy: a problematic channel? BJU Int 2001; 87: 861-865.

18 Casale AJ. A long continent ileovesicostomy using a single piece of bowel. J Urol 1999; 162: 1743-1745.

19 Koyle MA, Mingin GC, Furness III PD, Malone PS. The Mitrofanoff (flap valve principle): Application in contemporary continent urinary and gastrointestinal reconstruction. AUA Update Ser 2004; 23: 273-279.

20 Castellan M, Gosalbez R, Labbie A, Ibrahim E, Disandro M. Bladder neck sling for treatment of neurogenic incontinence in children with augmentation cystoplasty: long-term followup. $J$ Urol 2005; 173: 2128-2131; discussion 2131.

21 Madersbacher S, Schmidt J, Eberle JM, Thoeny HC, Burkhard F, Hochreiter W et al. Long-term outcome of ileal conduit diversion. J Urol 2003; 169: 985-990.

22 Khoury JM, Timmons SL, Corbel L, Webster GD. Complications of enterocystoplasty. Urology 1992; 40: 9-14. 